\title{
Spectral and entropy changes for back muscle fatigability following spinal stabilization exercises
}

\author{
Tae-Ro Lee, PhD; ${ }^{1}$ Yoon Hyuk Kim, PhD; ${ }^{2}$ Paul S. Sung, PhD, DHSc, PT ${ }^{3^{*}}$ \\ ${ }^{1}$ Department of Healthcare Management, College of Health Science, Korea University, Seoul, Korea; ${ }^{2}$ Department of \\ Mechanical Engineering, Kyung Hee University, Yongin, Korea; ${ }^{3}$ Department of Physical Therapy, College of Health \\ Science, Korea University, Seoul, Korea
}

\begin{abstract}
The entropy of electromyography (EMG) signals suggests a possible tool for the clinical assessment of low back pain (LBP). However, a general physiological mechanism for entropy and pain and/or dysfunction following exercise intervention is still unknown. This comparative study investigated the differences between entropy levels of the EMG signals and the slope of median frequency (MF) based on power spectrum analysis in chronic LBP subjects after 4 weeks of spinal stabilization exercises (SSEs). In total, 46 subjects (24 female and 22 male) participated in the exercise program; the EMG signals of their thoracic and lumbar erector spinae muscles were measured. Following the SSEs, the level of pain as measured by the Million Visual Analog Scale decreased significantly from 3.80 to 2.81 ( $T=3.42, p=0.001$ ). The Shannon entropy levels of the EMG signals were not different based on the back muscles $(F=2.86, p=0.09$ ) but demonstrated a significant interaction with pain level $(F=7.17, p=0.01)$. The slope of MF differed among the muscles $(F=12.06, p=0.01$ ); however, no interaction with the level of pain was found $(F=$ $0.19, p=0.66$ ). Therefore, the Shannon entropy of the EMG signals might be a useful and valuable clinical tool to measure pain following intervention.
\end{abstract}

Key words: complexity, EMG, entropy, erector spinae, low back pain, Million Visual Analog Scale, power spectrum, rehabilitation, slope of median frequency, spinal stabilization exercises.

\section{INTRODUCTION}

Several studies have reported that entropic measures for surface electromyography (EMG) time series are useful for evaluating localized muscular fatigue in a noninvasive fashion [1-5]. A previous study confirmed that the entropy measurement for characterizing neuromuscular alterations is reliable [6]. As a result, Shannon entropy, which measures complexity and the degree of uncertainty of information, could be widely utilized to assess localized muscle fatigability.

Numerous studies have identified an association between easily fatigued back muscles and low back pain (LBP) based on endurance tests [7-9]. However, no data have determined whether exercises can alter erector spinae (ES) muscle fatigue in subjects with chronic LBP.

\footnotetext{
Abbreviations: ANOVA = analysis of variance, $\mathrm{EMG}=$ electromyography, ES = erector spinae, FFT = fast Fourier transformation, LBP = low back pain, MF = median frequency, MVAS = Million Visual Analog Scale, SE = standard error, SSE = spinal stabilization exercise.

*Address all correspondence to Paul S. Sung, PhD, DHSc, PT; Department of Physical Therapy, College of Health Science, Korea University, 1 Jeongneung 3-dong, Sungbukgu, Seoul, Korea 136-703; +82-2-940-2831; fax: +82-2-9402830. Email: psung@korea.ac.kr

DOI:10.1682/JRRD.2009.07.0088
} 
A lack of cocontraction of the lumbar ES muscles might be a significant factor in chronic LBP [10-11]. Spinal stabilization exercises (SSEs) improve the cocontraction of trunk muscles, which may restore stability to the spine and, theoretically, may protect it from biomechanical stresses and further injuries [12].

The time-dependent entropy of the EMG signal can be analyzed in several ways. One of the analyses, Shannon entropy, is a standard measure of complexity and has been applied in research in cognitive science, aging, heart failure, and other fields [4,13-14]. We found in a previous study that the plateau value of the entropy was lower for subjects with LBP than for control subjects. This connection might prove useful in a clinical assessment of LBP. It is important to understand that Costa et al. and Chialvo argued that pathology and/or dysfunction is associated with less variability [1-3]. Generally, biological time series are complex data that need to be distilled to useful application to assess a pathology and/or dysfunction. In our current study, the entropy levels of the EMG signals and median frequency (MF) slopes of the EMG signals were reported.

A connection exists between localized muscular fatigue and EMG spectral analyses for back muscle fatigue following intervention [15-17]. Several studies have been applied to a variety of physiological time series, such as human heartbeats [13,18-19]. The degree of randomness is possibly a characteristic property of time series. For example, variations in heart rate could be considered healthy, whereas a very steady heart rate is generally associated with disease. Thus, it would be important to compare Shannon entropy levels of the EMG signal and spectral quantities following intervention with a level of musculoskeletal pain.

Several other studies have compared entropy levels of the EMG for subjects with and without illness and/or dysfunction [14,19-22]. However, no study has investigated pain-level changes following specific therapeutic intervention in subjects with chronic LBP. People with chronic LBP often have decreased muscle endurance, which may compromise the functional capacity of the spine and increase the likelihood of reinjury [23]. Numerous studies have also identified an association between LBP and easily fatigued back muscles based on back muscle endurance [8-9,24-26]. However, no data are available as to whether SSE is capable of altering patterns of localized muscle fatigue in chronic LBP.
Subjects with LBP have also been reported to have less endurance, and thus smaller MF slopes, during sustained muscle contractions [17,27-28]. The signal from conventional surface EMG is the instantaneous algebraic summation of action potentials from muscle fibers, and its power spectrum can be estimated from a fast Fourier transformation (FFT) of the signal. The MF is calculated from the spectrum and reflects, in part, the propagation speed of the action signal. However, the MF based on the FFT still remains elusive because of conflicting results. It would be valuable to confirm the measurements following SSE for characterizing neuromuscular alterations through investigation of differences between the power spectrum analysis and nonlinear time-series analysis of entropy measures. Therefore, the purpose of this study was to assess the effects of 4 weeks of SSEs based on entropic measures of ES muscle fatigability compared with the slope of the MF based on power spectrum analyses in subjects with chronic LBP. Demographic factors were also considered in the determination of those outcome variables.

\section{METHODS}

\section{Selection of Subjects}

The focus of this study was back muscle fatigability, especially the thoracic and lumbar ES muscles, in subjects experiencing LBP. Subjects in this study were defined as those who presented with LBP, met study inclusion criteria, and had experienced a disturbing impairment or abnormality in the functioning of the low back for more than 2 months [29].

Subjects were eligible to participate if they (1) were 21 years of age or older and (2) had LBP for more than 2 months without pain referral into the lower limbs. Individuals were excluded from participation if they (1) had a diagnosed psychological illness that might interfere with the study protocol, (2) had experienced overt neurological signs (sensory deficits or motor paralysis), or (3) were pregnant. Subjects were withdrawn from the study if they requested to withdraw. Those subjects who met study inclusion criteria received information regarding the purpose and methods of the study and signed a copy of the institutional review board-approved consent form. 


\section{Pain Level}

Subject disability was inferred from self-reported scores on the Million Visual Analog Scale (MVAS) of pain interference, which was given to each subject during the initial and final testing sessions. The MVAS includes 15 questions that subjects answer by marking a point on a $10 \mathrm{~cm}$ line anchored at each end. Responses range from 0 to $10 \mathrm{~cm}$, where $10 \mathrm{~cm}$ represents maximum disability.

\section{Electromyography Measurement}

For all subjects, EMG measurements were obtained under identical conditions before and after the 4-week SSE program. No subjects underwent any traumatic event or injury between the two measurement days.

In this study, the endurance of the back muscles was determined by a modified version of the isometric fatigue test from our previous study [2]. The subjects' upper bodies were positioned with their iliac crests at the edge of the table; their lower bodies were secured at the ankle and hamstring levels with seatbelt straps. Subjects held their arms across their chests with each hand placed on the opposite shoulder, and standard verbalized encouragement was given throughout the test.

The EMG electrodes were placed bilaterally over the greatest convexity of the thoracic ES muscles at the T10 T11 level and the lumbar ES muscles at the L4-L5 level with a $10 \mathrm{~cm}$ distance between electrodes of each pair. The electrode sites and the distance of the electrodes were carefully determined for each subject according to Zipp [30]. The EMG signals were preamplified at the skin (gain 35×) and further amplified downstream (bandwidth 20-4,000 Hz; model D-100 preamplifier and model ENG 55 driver amplifier, Therapeutics Unlimited; Iowa City, Iowa) with the total system adjusted for each subject to allow maximal amplification without saturation of the analog-to-digital converter. The EMG signal was fed through a low-pass filter (cutoff frequency $480 \mathrm{~Hz}$ at $6 \mathrm{~dB}$ per octave) and subsequently passed to a BNC connector board (BNC 2080, National Instruments; Austin, Texas). The signal also interfaced with a 12-bit analog-todigital converter (AT-MIO-16E-10, National Instruments) that amplified 100 $\times$ and sampled each channel at $1,024 \mathrm{~Hz}$. The digitized data were stored on computer disks for subsequent analysis.

Using standard FFT of the EMG data, we obtained the power spectrum for each 1-second time interval. The MF of the signal, $\langle f\rangle$, was calculated from the spectrum for each 1-second time interval.
The EMG signals from the isometric fatigue test were transformed into their frequency spectrum by a wavelet analysis. The MF of the spectrum was then recorded. After dividing the range of $X(t)$ into 500 equalsized bins, we used a histogram to determine the probability distribution $p_{j, t}$. The entropy is calculated from the Equation

$$
S_{t}=-\sum_{j} p_{j, t} \ln p_{j, t}
$$

where $p_{j}$ is the probability for outcome number $j$ of a given experiment [20-23]. This equation is the standard formulation of uncertainty because it has the following features: (1) the lowest entropy $(S=0)$ corresponds to one of the outcomes being certain (i.e., probability one) and the others never occurring (i.e., probability zero), (2) the largest value for the entropy $(S=\ln M)$ is achieved when all outcomes are equally likely (all probabilities are equal to each other $p_{j}=1 / M$ ), and (3) $S$ is additive over partitions of the outcomes [2].

The entropy $S$ versus time exhibits a plateau for $t>$ $10 \mathrm{~ms}$. This plateau value of $S_{t}$ was referred to as the entropy. The detailed entropy calculation process is fully described in previous studies [1-3]. Our previous data were partially used in this study in order to monitor the effects of the exercise intervention.

\section{Spine Stabilization Exercise Protocol}

The subjects came to the laboratory once a week for 4 weeks in addition to performing the exercises at home daily; the subjects were supervised in the laboratory to ensure they were performing the exercises correctly. The SSE approach used in this study is commonly advocated in the rehabilitation of LBP patients [17,31]. For example, the quadruped exercise (Table 1), performed from an all-fours position with the arms and legs extending reciprocally, is used to recruit the trunk and hip extensors. The exercise program consisted of five different types of exercises, such as upper-body extension in prone position, alternate arm and leg lifts in quadruped position, alternate arm and leg lifts in prone position, and diagonal curl up and straight curl up in supine position; the exercise program was done daily for 20 minutes. To ensure adherence, the subjects kept an exercise log and received telephone calls at least once a week. The intensity of the exercises was at the subject's tolerance level, and the subjects were encouraged to report any problems immediately. 
JRRD, Volume 47, Number 2, 2010

Table 1.

Spinal stabilization exercise program for subjects with lower back pain.

\begin{tabular}{cl}
\hline \multicolumn{1}{c}{ Type of Exercise } & \multicolumn{1}{c}{ Description } \\
\hline Upper-Body Extension & With pillow supporting abdo- \\
& men, clasp hands behind back \\
& and lift body off floor. Keep \\
chin tucked while lifting in & prone position.
\end{tabular}

Alternate Arm and Leg Lift $\quad$ Keep knee locked and lift leg 8-10 in. from floor along with opposite arm in quadruped position.

Alternate Arm and Leg Extension on All Fours

Diagonal Curl Up

Curl Up
Raise opposite arm and leg in quadruped position. Do not arch neck.

Keeping arms folded across chest, tilt pelvis to flatten back in supine position. Lift head and shoulders from floor while rotating to one side.

With arms at sides, tilt pelvis to flatten back. Raise shoulders and head from floor in supine position. Use arms to support trunk if necessary.

\section{Statistical Analysis}

Descriptive statistics were used to compare the mean and standard deviation of each muscle group as well as subject characteristics. The entropy levels of the EMG signals and MF slopes for the thoracic and lumbar ES muscles were compared. The repeated measure analysis of variance (ANOVA) was used to examine any significant differences between power spectrum analysis for MF slope and entropy levels of the EMG signals based on nonlinear time series before and after the intervention. Any continuous variables, including the level of pain variable, were used as covariates to eliminate confounding effects.

When their joint effect is discernibly larger or smaller than the "sum of the parts," we say that there is an "interaction" among the explanatory variables [32]. When two or more explanatory variables are considered simultaneously, one could ask whether their joint effect on the response variable is simply the sum of the parts (or individual effects). In our study, the factorial designs

were used to investigate the main effect, which is what the independent variables elicit when averaged out over each other. Regarding the design of experiments and ANOVA, a main effect is the effect of an independent variable on a dependent variable averaging across the levels of any other independent variables. Therefore, the level of pain was considered with regard to both outcome variables (entropy level and slope of MF).

The entropy value of the EMG signals was analyzed to compare the differences between pre- and postexercise intervention in subjects with LBP. The MathCad package (MathSoft; Cambridge, Massachusetts) was used for this analysis, which was loaded onto a personal computer running the Windows XP operating system (Microsoft Corp.; Redmond, Washington).

Assumptions of repeated measures, including homogeneity of variance, normal distribution of data, and sphericity, were tested with Mauchly's test [33]. If the assumption of sphericity was violated, Greenhouse-Geisser adjustments to the degrees of freedom were applied [33]. For all statistical tests, type I error rate was set at 0.05 .

\section{RESULTS}

The subjects' demographics are summarized in Table 2. Forty-six subjects (22 males and 24 females) participated in the study. Overall, no significant sex differences based on age ( $t=0.11, p=0.91)$, number of months since pain onset $(t=-0.48, p=0.63)$, or MVAS score $(t=0.24, p=0.80)$ were found. Following the exercise intervention, the average level of pain decreased significantly from 3.80 to $2.81(T=3.42, p=0.001)$. Therefore, the level of pain was used as a covariate to control confounding effects for both outcome variables (entropy of the EMG signals and the slope of the MF).

The results for the thoracic and lumbar ES muscles based on the entropy levels of the EMG signals and MF slope are illustrated in Figures $\mathbf{1}$ and 2. The mean entropy levels of the EMG signals \pm standard error were analyzed pre- and postexercises. Differences in the levels of the right thoracic ES muscle $(1.76 \pm 0.06$ vs $1.70 \pm$ 0.07), left thoracic ES muscle (1.19 \pm 0.05 vs $1.12 \pm$ $0.05)$, right lumbar ES muscle (1.46 \pm 0.06 vs $1.45 \pm$ $0.08)$, and left lumbar ES muscle (1.75 \pm 0.06 vs $1.67 \pm$ 0.06) were evident (Figure 1). The Shannon entropy levels of the EMG signals were not different based on the back muscles $(F=2.86, p=0.09)$; however, the entropy 
Table 2.

Demographics of study participants with chronic low back pain.

\begin{tabular}{|c|c|c|c|c|}
\hline Variable & Male $(n=22)$ & Female $(n=24)$ & $t$-Value & $p$-Value \\
\hline Age (yr) & & & 0.68 & 0.49 \\
\hline Range & $36-64$ & $26-64$ & & \\
\hline Months Since Pain Onset & & & 0.11 & 0.91 \\
\hline Range & $4-24$ & $4-17$ & & \\
\hline Range & $1.31-7.90$ & $1.35-5.46$ & & \\
\hline Mean \pm SD & $3.75 \pm 1.50$ & $3.84 \pm 1.00$ & & \\
\hline
\end{tabular}

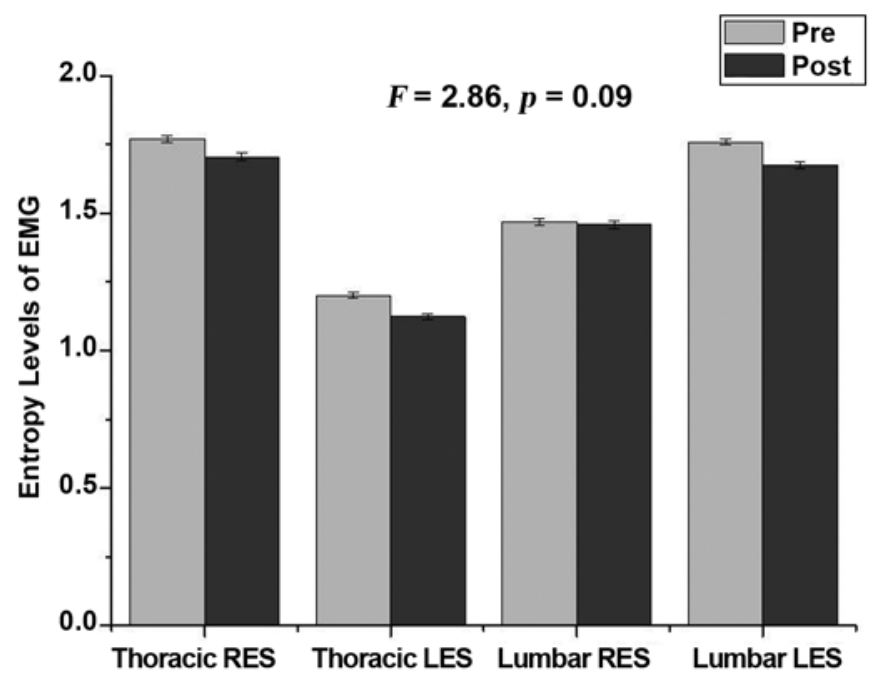

Figure 1.

Shannon entropy measurements (mean \pm standard error) for both sides of erector spinae (ES) muscle pre- and postintervention. Entropy levels of electromyography (EMG) decreased following spinal stabilization exercises for both sides of ES muscle (RES = right ES, LES = left ES).

demonstrated a significant interaction with pain level $(F=$ $7.17, p=0.01)$. In addition, other demographic variables, such as age $(F=0.86, p=0.35)$ and $\operatorname{sex}(F=0.99, p=$ 0.32 ), had no interaction with the entropy levels of the EMG signals.

In Figure 2, the MF slopes of the EMG signals \pm SE were analyzed pre- and postexercises. Differences in preand post-MF slope levels of the right thoracic ES muscle $(-0.17 \pm 0.02$ vs $-0.19 \pm 0.02)$, left thoracic ES muscle $(-0.19 \pm 0.01$ vs $-0.17 \pm 0.02)$, right lumbar ES muscle

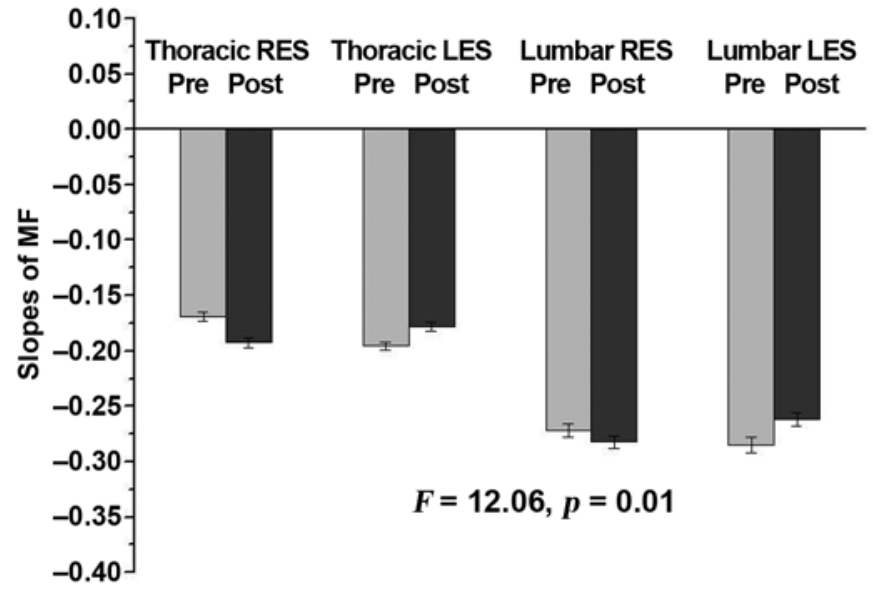

Figure 2.

Slope of median frequency (MF) measurements (mean \pm standard error) for both sides of erector spinae (ES) muscle pre- and postintervention. Lumbar portion of ES muscle demonstrated steeper slope levels of MF than thoracic muscles (RES = right ES, LES = left ES).

$(-0.27 \pm 0.03$ vs $-0.28 \pm 0.03)$, and left lumbar ES muscle $(-0.28 \pm 0.04$ vs $-0.26 \pm 0.03)$ were evident. The slope of MF based on power spectrum analysis was different between muscles $(F=12.06, p=0.01)$; however, the MF did not demonstrate any interactions with level of pain $(F=$ $0.19, p=0.66)$ or exercise intervention $(F=0.08, p=$ $0.77)$. The other demographic variables, such as age $(F=$ $0.32, p=0.57)$ and sex $(F=12.06, p=0.20)$, had no interaction with MF slopes of the EMG.

As shown in Figure 3, positive correlations based on the lumbar and thoracic ES muscles were evident. The thoracic portion of the ES muscle demonstrated a relatively greater positive association $(r=0.86, p<0.01)$ 
than the lumbar muscles $(r=0.76, p<0.01)$. No significant correlation existed between entropy levels of the EMG signals and the slope of MF with pain level following the intervention.

\section{DISCUSSION}

This study assessed the effects of 4 weeks of exercise intervention based on entropic measures of the EMG signals from both sides of the thoracic and lumbar ES mus-

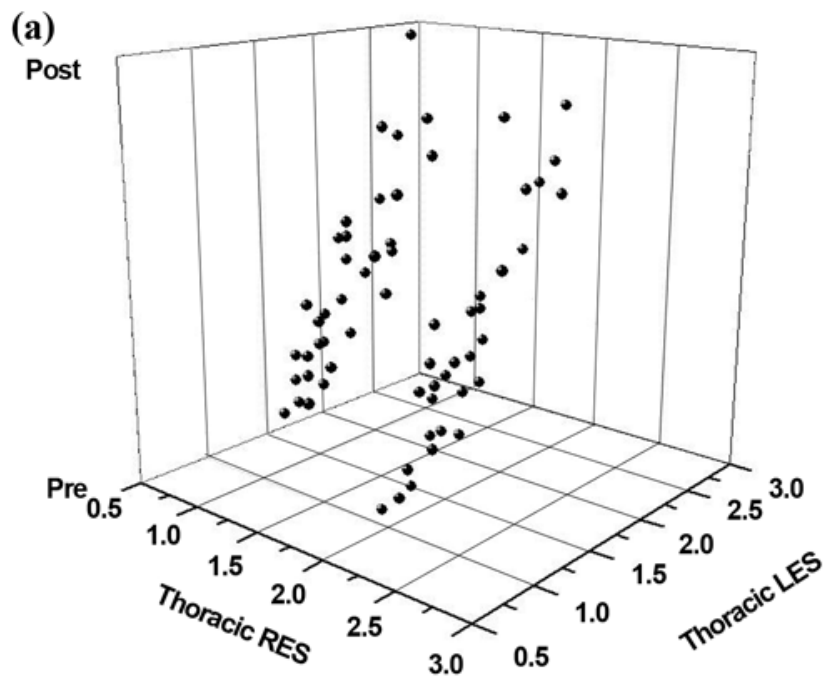

(b)

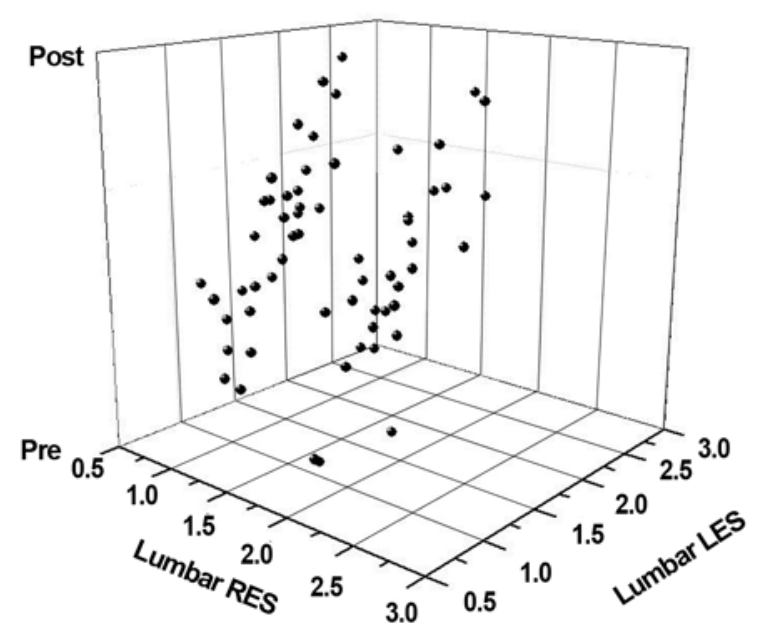

Figure 3.

Pearson correlation analyses of entropy levels of electromyography for both sides of erector spinae (ES) muscle before (Pre) and after (Post) 4 weeks of spine stabilization exercise. (a) Thoracic portion of ES muscle demonstrated relatively greater positive association ( $r=$ 0.86, $p<0.01)$ than (b) lumbar muscles $(r=0.76, p<0.01)$. RES $=$ right $\mathrm{ES}, \mathrm{LES}=$ left ES. cles compared with power spectrum analyses. Following the intervention, the level of pain reported by the subjects decreased significantly. The Shannon entropy levels of the EMG signals decreased and significantly interacted with pain level. However, the slope levels based on power spectrum analysis did not demonstrate any interaction with the level of pain, although the slopes of MF (two muscles among four muscles) decreased following the completion of the exercise program.

The term "interaction" has a very precise statistical meaning and refers to how the effect on the response of one explanatory variable depends on the level of one or more other explanatory variables [32]. The general definition of interaction implies that if no interaction exists between two explanatory variables, then the effect of one explanatory variable is constant or remains the same across all levels or values of the other. If the effect of one factor depends on the level of another factor, the two factors involved are said to interact and a contrast involving all these levels is called their interaction [32]. The results of our study indicated that the level of pain depends on the response of entropy changes. Therefore, the effect of the level of pain has an interaction with and depends on another explanatory variable, which is entropy change among muscles.

Our previous study indicated that control subjects revealed significantly larger entropy levels of the EMG signals than subjects with LBP [3]. Thus, the results of the current study consistently demonstrated a connection between physiological "health" and complexity $[4,13]$. We also explored the use of entropy derived from time series as an alternative quantitative measure of EMG signals that can be used in a clinical assessment [3]. The entropy levels of the EMG signals decreased following the intervention.

The results of our study also indicated that Shannon entropy levels of the EMG signals were not different based on the back muscles ( $F=2.86, p=0.09$ ). However, the entropy levels of the EMG signals significantly interacted with the level of pain. The side difference of the back muscles could be related to dominance or other physiological reasons. The participants in our study were all right-hand dominant; therefore, the left side of the back was considered the dominant side, as our previous study indicated [34]. If other studies did not consider the dominant side of the back in this way, the results may be misinterpreted in conjunction with side assessed and result in confounding effects. 
The results of our current study indicated that the entropy levels of the right thoracic and left lumbar muscles were higher, although the entropy levels did not statistically differ. As indicated in Figure 2, the right thoracic and left lumbar muscles demonstrated higher entropy levels. This pattern might be necessary to maintain the Sorensen testing position; therefore, further investigation based on the handedness of the subjects is warranted, since muscle development could be related to LBP. Although no significant entropy-level difference was seen, the entropy level of the thoracic ES was higher on the right side than on the left side. Both sides of the thoracic and lumbar trunk muscles were also different, and this difference was significant when the exercise intervention was considered.

The results of the study indicated no statistical difference following the intervention to affect entropy levels of the EMG signals or the slope of the MF. Previous studies have assessed EMG of the back muscles of subjects with LBP undergoing rehabilitation [8-9,24-26,35]. However, no data are available as to whether SSEs are capable of altering patterns of muscle fatigue in chronic LBP subjects. SSEs involve the cocontraction of muscles, which may restore stability to the spine and theoretically protect it from biomechanical stresses and further injuries [36]. According to Janda, the ES muscles stiffen more than the abdominal muscles in subjects with LBP [37]. A 4-week exercise intervention significantly reduced LBP; however, the overall change in entropy levels of the EMG signals and the slope of MF were not sensitive enough to demonstrate a significant change given the limited exercise period. Therefore, the exercise intervention may affect back muscle function by mechanisms other than improvement in endurance of the stabilizing musculature.

The MF results also indicated that the muscle fiber action potential propagates at a slower velocity and undergoes an alteration in shape due to changes in the excitability of the muscle cell membrane during a sustained contraction. This pattern is typically measured during a contraction as a decrease in the MF of the EMG signal. Individuals with better endurance exhibit a less precipitous decay rate of the MF $[14,28]$. The differences may not be detected either because the measurement was not sensitive enough or because physiological changes did not occur [38].

Another important finding of our study indicated that the entropy levels of the EMG signals demonstrated significant interaction with pain level. However, the slope of the MF did not demonstrate this interaction. The results of this study indicated that subjects with LBP demonstrated higher fatigability of the ES muscles regardless of sex. No significant relationship existed between the MF of the ES muscles and demographic variables, such as sex and age, when the level of pain was controlled as a covariate.

Muscle biopsy analyses showed that the thoracic and lumbar regions of the back muscles suggested that the ratio of type I to type II muscle fibers in a cross-sectional area is significantly greater in women than in men [28]. Again, our study did not support back muscle endurance differences between males and females.

The significant interaction effect between entropy and pain level for muscle endurance during the 1-minute back-extension test supports the change in the frequency characteristics of the recorded signals that occurs with fatigue [39]. Exercises for graded activity programs can be used to increase trunk muscle endurance and decrease pain [40-41]. Undoubtedly, other muscles participated in the load sharing during the testing as well as when subjects performed the intervention exercises. The attachment of the lumbar ES muscles, rather than the thoracic ES muscles, results in an effective lever arm for lumbar stabilization. Therefore, the lumbar ES muscle is more effective in creating a stabilizing moment over the lumbar vertebral segments during the test [42-43].

The results of the study indicated a significant interaction between entropy levels of the EMG signals and level of pain following stabilization exercises. However, the level of pain did not demonstrate interactions with the slopes of MF. Therefore, the results of this study indicated that Shannon entropy might be a valuable tool to measure the level of pain following the exercise intervention. Future studies might include a larger sample size and control groups to generalize the results. Follow-up, randomized controlled trials to more fully investigate treatment effects and factors that might mediate these effects should be pursued.

\section{CONCLUSIONS}

This study investigated back muscle fatigability following exercise intervention in subjects with chronic LBP. The results of this study indicated that the average pain levels reported by the subjects decreased significantly following 4 weeks of an SSE program. The entropy decreased as well and was found to have a significant 
interaction with pain level. The slope of MF based on power spectrum analysis also decreased but did not demonstrate any interaction with pain level. Therefore, the results of this study indicated that Shannon entropy might reveal valuable information regarding pain levels following intervention.

\section{ACKNOWLEDGMENTS}

\author{
Author Contributions: \\ Study concept and design: T.-R. Lee. \\ Administrative and technical support; fund procurement: Y. H. Kim. \\ Research design, data collection, project management, data analysis, \\ drafting and revision of manuscript: P. S. Sung. \\ Additional Contributions: We also thank Drs. Miron Kaufman and \\ Ulrich Zurcher of Cleveland State University, Cleveland, Ohio, for \\ their critical analyses of the important intellectual content and inter- \\ pretation of data. \\ Financial Disclosures: The authors have declared that no competing \\ interests exist. \\ Funding/Support: This material was based on work supported by the \\ Korea University and the National Agenda Project funded by the \\ Korea Research Council of Fundamental Science and Technology. \\ Institutional Review: This study was approved by the Cleveland \\ State University Institutional Review Board. Subjects received infor- \\ mation about the study and signed a copy of the institutional review \\ board-approved consent form.
}

Participant Follow-Up: The authors do not plan to inform participants of the publication of this study.

\section{REFERENCES}

1. Kaufman M, Zurcher U, Sung P. Entropy of electromyography time series. Physica A. 2007;386(2):698-707.

2. Sung PS, Zurcher U, Kaufman M. Nonlinear analysis of electromyography time series as a diagnostic tool for low back pain. Med Sci Monit. 2005;11(1):CS1-5.

[PMID: 15614197]

3. Sung PS, Zurcher U, Kaufman M. Comparison of spectral and entropic measures for surface electromyography time series: A pilot study. J Rehabil Res Dev. 2007;44(4):599-609. [PMID: 18247257] DOI:10.1682/JRRD.2006.10.0132

4. Costa M, Goldberger AL, Peng CK. Multiscale entropy analysis of biological signals. Phys Rev E Stat Nonlin Soft Matter Phys. 2005;71(2 Pt 1):021906. [PMID: 15783351$]$ DOI:10.1103/PhysRevE.71.021906

5. Aho AJ, Yli-Hankala A, Lyytikäinen LP, Jäntti V. Facial muscle activity, Response Entropy, and State Entropy indices during noxious stimuli in propofol-nitrous oxide or propofol-nitrous oxide-remifentanil anaesthesia without neuromuscular block. Br J Anaesth. 2009;102(2):227-33.
[PMID: 19112059]

DOI:10.1093/bja/aen356

6. Sung PS, Zurcher U, Kaufman M. Reliability difference between spectral and entropic measures of erector spinae muscle fatigability. J Electromyogr Kinesiol. 2010;20(1):25-30. [PMID: 19121587] DOI:10.1016/j.jelekin.2008.11.005

7. McGill SM. Low back exercises: Evidence for improving exercise regimens. Phys Ther. 1998;78(7):754-65. [PMID: 9672547]

8. Mayer TG, Kondraske G, Mooney V, Carmichael TW, Butsch R. Lumbar myoelectric spectral analysis for endurance assessment. A comparison of normals with deconditioned patients. Spine (Phila Pa 1976). 1989;14(9):986-91. [PMID: 2781412] DOI:10.1097/00007632-198909000-00013

9. Klein AB, Snyder-Mackler L, Roy SH, DeLuca CJ. Comparison of spinal mobility and isometric trunk extensor forces with electromyographic spectral analysis in identifying low back pain. Phys Ther. 1991;71(6):445-54. [PMID: 1827921]

10. Cholewicki J, Juluru K, Radebold A, Panjabi MM, McGill SM. Lumbar spine stability can be augmented with an abdominal belt and/or increased intra-abdominal pressure. Eur Spine J. 1999;8(5):388-95. [PMID: 10552322] DOI:10.1007/s005860050192

11. Panjabi MM. The stabilizing system of the spine. Part I. Function, dysfunction, adaptation, and enhancement. J Spinal Disord. 1992;5(4):383-89. [PMID: 1490034] DOI:10.1097/00002517-199212000-00001

12. Richardson CA, Snijders CJ, Hides JA, Damen L, Pas MS, Storm J. The relation between the transversus abdominis muscles, sacroiliac joint mechanics, and low back pain. Spine (Phila Pa 1976). 2002;27(4):399-405. [PMID: 11840107] DOI:10.1097/00007632-200202150-00015

13. Costa M, Goldberger AL, Peng CK. Multiscale entropy analysis: A new measure of complexity loss in heart failure. J Electrocardiol. 2003;36 Suppl:39-40.

DOI:10.1016/j.jelectrocard.2003.09.011

14. Goldberger AL, Amaral LA, Hausdorff JM, Ivanov PCh, Peng CK, Stanley HE. Fractal dynamics in physiology: Alterations with disease and aging. Proc Natl Acad Sci U S A. 2002;99 Suppl 1:2466-72. [PMID: 11875196]

DOI:10.1073/pnas.012579499

15. Mannion AF, Knecht K, Balaban G, Dvorak J, Grob D. A new skin-surface device for measuring the curvature and global and segmental ranges of motion of the spine: Reliability of measurements and comparison with data reviewed from the literature. Eur Spine J. 2004;13(2):122-36.

[PMID: 14661104]

DOI:10.1007/s00586-003-0618-8 
16. Mannion AF, Müntener M, Taimela S, Dvorak J. Comparison of three active therapies for chronic low back pain: Results of a randomized clinical trial with one-year followup. Rheumatology (Oxford). 2001;40(7):772-78.

[PMID: 11477282]

DOI:10.1093/rheumatology/40.7.772

17. Sung PS. Multifidi muscles median frequency before and after spinal stabilization exercises. Arch Phys Med Rehabil. 2003;84(9):1313-18. [PMID: 13680467]

DOI:10.1016/S0003-9993(03)00139-4

18. Ivanov PC, Amaral LA, Goldberger AL, Havlin S, Rosenblum MG, Struzik ZR, Stanley HE. Multifractality in human heartbeat dynamics. Nature. 1999;399(6735):461-65.

[PMID: 10365957]

DOI:10.1038/20924

19. Costa MG, Goldberger AL, Peng CK. Multiscale entropy analysis of complex physiologic time series. Phys Rev Lett. 2002;89(6):068102. [PMID: 12190613]

DOI:10.1103/PhysRevLett.89.068102

20. Stanley HE, Buldyrev SV, Goldberger AL, Hausdorff JM, Havlin S, Mietus J, Peng CK, Sciortino F, Simons M. Fractal landscapes in biological systems: Long-range correlations in DNA and interbeat heart intervals. Physica A. 1992;191(1-4):1-12. [PMID: 11537103] DOI:10.1016/0378-4371(92)90497-E

21. Chialvo DR. Physiology: Unhealthy surprises. Nature. 2002;419(6904):263. [PMID: 12239553]

DOI:10.1038/419263a

22. West B. Fractal physiology and chaos in medicine. Singapore: World Scientific; 1990.

23. Jackson CP, Brown MD. Is there a role for exercise in the treatment of patients with low back pain? Clin Orthop Relat Res. 1983(179):39-45. [PMID: 6225594$]$

24. McGill SM. Low back exercises: Evidence for improving exercise regimens. Phys Ther. 1998;78(7):754-65. [PMID: 9672547]

25. Roy SH, De Luca CJ, Emley M, Buijs RJ. Spectral electromyographic assessment of back muscles in patients with low back pain undergoing rehabilitation. Spine (Phila $\mathrm{Pa}$ 1976). 1995;20(1):38-48. [PMID: 7709278]

DOI:10.1097/00007632-199501000-00008

26. Biedermann HJ, Shanks GL, Forrest WJ, Inglis J. Power spectrum analyses of electromyographic activity. Discriminators in the differential assessment of patients with chronic low-back pain. Spine (Phila Pa 1976). 1991;16(10): 1179-84. [PMID: 1836678]

27. Roy SH, De Luca CJ, Emley M, Oddsson LI, Buijs RJ, Levins JA, Newcombe DS, Jabre JF. Classification of back muscle impairment based on the surface electromyographic signal. J Rehabil Res Dev. 1997;34(4):405-14.

[PMID: 9323644]
28. Mannion AF, Connolly B, Wood K, Dolan P. The use of surface EMG power spectral analysis in the evaluation of back muscle function. J Rehabil Res Dev. 1997;34(4):427-39. [PMID: 9323646]

29. Klenerman L, Slade PD, Stanley IM, Pennie B, Reilly JP, Atchison LE, Troup JD, Rose MJ. The prediction of chronicity in patients with an acute attack of low back pain in a general practice setting. Spine (Phila Pa 1976). 1995;20(4): 478-84. [PMID: 7747233]

30. Zipp P. Recommendations for the standardization of lead positions in surface electromyography. Eur J Appl Physiol. 1982;50:41-54. DOI:10.1007/BF00952243

31. O’Sullivan P, Twomey L, Allison G, Sinclair J, Miller K. Altered patterns of abdominal muscle activation in patients with chronic low back pain. Aust J Physiother. 1997;43(2): 91-98. [PMID: 11676676]

32. Fitzmaurice G. The meaning and interpretation of interaction. Nutrition. 2000;16(4):313-14. [PMID: 10758374 DOI:10.1016/S0899-9007(99)00293-2

33. Kleinbaum DG, Kupper LL, Muller KE. Applied regression analysis and other multivariable methods. Belmont (CA): Duxbury Press; 1988. p. 11-15.

34. Sung PS, Spratt KF, Wilder DG. A possible methodological flaw in comparing dominant and nondominant sided lumbar spine muscle responses without simultaneously considering hand dominance. Spine (Phila Pa 1976). 2004;29(17): 1914-22. [PMID: 15534417$]$ DOI:10.1097/01.brs.0000137071.47606.19

35. Chok B, Lee R, Latimer J, Tan SB. Endurance training of the trunk extensor muscles in people with subacute low back pain. Phys Ther. 1999;79(11):1032-42.

[PMID: 10534796$]$

36. Richardson CA, Jull GA. Muscle control-pain control. What exercises would you prescribe? Man Ther. 1995;1(1): 2-10. [PMID: 11327788] DOI:10.1054/math.1995.0243

37. Janda V. Muscles, central nervous motor regulation and back problems. In: Korr IM, editor. The neurologic mechanisms in manipulative therapy. New York (NY): Plenum Press; 1978.

38. Moffroid MT, Haugh LD, Haig AJ, Henry SM, Pope MH. Endurance training of trunk extensor muscles. Phys Ther. 1993;73(1):10-17. [PMID: 8417455$]$

39. Roy SH, De Luca CJ, Casavant DA. Lumbar muscle fatigue and chronic lower back pain. Spine (Phila Pa 1976). 1989;14(9):992-1001. [PMID: 2528828]

DOI:10.1097/00007632-198909000-00014

40. Jørgensen K, Nicolaisen T. Two methods for determining trunk extensor endurance. A comparative study. Eur J Appl Physiol Occup Physiol. 1986;55(6):639-44.

[PMID: 2946578] DOI:10.1007/BF00423210 
41. Jørgensen K, Nicolaisen T. Trunk extensor endurance: Determination and relation to low-back trouble. Ergonomics. 1987;30(2):259-67. [PMID: 2953591]

DOI:10.1080/00140138708969704

42. MacIntosh JE, Bogduk N. The biomechanics of the lumbar multifidus. Clin Biomech. 1986;1:205-13.

DOI:10.1016/0268-0033(86)90147-6

43. Flicker PL, Fleckenstein JL, Ferry K, Payne J, Ward C, Mayer T, Parkey RW, Peshock RM. Lumbar muscle usage in chronic low back pain. Magnetic resonance image evaluation. Spine (Phila Pa 1976). 1993;18(5):582-86.

[PMID: 8484149]

DOI:10.1097/00007632-199304000-00010

Submitted for publication July 3, 2009. Accepted in revised form December 22, 2009. 